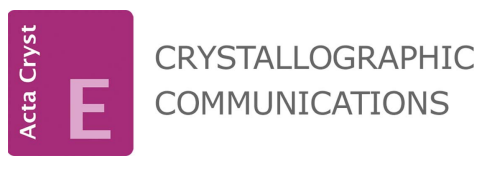

ISSN 2056-9890

Received 13 April 2018

Accepted 7 May 2018

Edited by H. Ishida, Okayama University, Japan

Keywords: crystal structure; arylmethyl dimedone; arylmethyl 1,3-cyclohexanedione; arylmethyl 3-hydroxycyclohex-2-enone.

CCDC reference: 1841730

Supporting information: this article has supporting information at journals.iucr.org/e

\section{Crystal structure of 3-hydroxy-2-(4-hydroxy-3- methoxyphenylmethyl)-5,5-dimethylcyclohex-2- enone}

\author{
Agnese Stikute, ${ }^{a}$ Karina Skestere, ${ }^{a}$ Inese Mierina, ${ }^{a}$ Anatoly Mishnev ${ }^{b} *$ and Mara \\ Jure $^{\mathrm{a} *}$
}

\begin{abstract}
anstitute of Technology of Organic Chemistry, Faculty of Materials Science and Applied Chemistry, Riga Technical University, P. Valdena Str. 3/7, Riga, LV-1048, Latvia, and ' ${ }^{\mathbf{b}}$ Latvian Institute of Organic Synthesis, Aizkraukles Str. 21, Riga, LV-1006, Latvia. *Correspondence e-mail: mishnevs@osi.Iv, Mara.Jure@rtu.Iv
\end{abstract}

In the title compound, $\mathrm{C}_{16} \mathrm{H}_{20} \mathrm{O}_{4}$, a new starting compound for the synthesis of various heterocycles, the partially saturated six-membered ring adopts a sofa conformation. An intramolecular $\mathrm{O}-\mathrm{H} \cdots \mathrm{O}$ hydrogen bond is observed in the guaiacol residue. In the crystal, molecules are assembled into a sheet structure parallel to the $a b$ plane via $\mathrm{O}-\mathrm{H} \cdots \mathrm{O}$ hydrogen bonds. The hydrogen-bond pattern is described by an $R_{4}^{4}(28)$ graph-set motif. The sheets are further linked by $\mathrm{C}-\mathrm{H} \cdots \mathrm{O}$ hydrogen bonds into a three-dimensional network.

\section{Chemical context}

Cyclic 2-arylmethyl-1,3-diketones attract interest as valuable intermediates for organic chemistry. A few of the latest examples of these cyclohexanedione derivatives have been used as starting compounds for the synthesis of various heterocycles [e.g. tetrahydrobenzofuranones (Yoshida et al., 2010) or tetrahydro-1H-xanthen-1-ones (Sudheendran et al., 2012)], as well as carbocycles, e.g. analogues of WielandMiesher and Hajos-Parrish ketones (Xu et al., 2013).<smiles>COc1cc(CC2=C(O)CC(C)(C)CC2=O)ccc1O</smiles>

$1 \mathrm{a}$

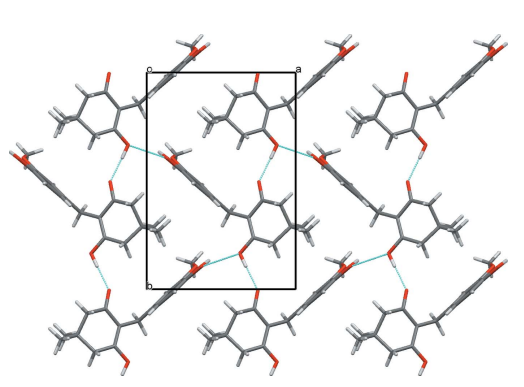

OPEN $\odot$ ACCESS

\section{Structural commentary}

Fig. 1 shows the molecular structure of the title compound, which exhibits an intramolecular $\mathrm{O}-\mathrm{H}$. . O hydrogen bond (Table 1). In crystalline state, the molecules assume the enol tautomeric form, 1a. In the dimedone fragment, the bond distances reflect the effect of conjugation in the flat fragment $\mathrm{O} 1=\mathrm{C} 3-\mathrm{C} 4=\mathrm{C} 5-\mathrm{O} 2$. The double bonds, $\mathrm{O} 1=\mathrm{C} 3$ and $\mathrm{C} 4=\mathrm{C} 5$, are elongated $[1.246$ (2) and 1.357 (3) $\AA$, respectively], while the single bond $\mathrm{C} 3-\mathrm{C} 4$ is shortened 
[1.447 (3) $\AA$ ] as compared with standard double and single bonds (Allen et al., 1987). The general shape of the molecule is characterized by the torsion angles $\mathrm{C} 3-\mathrm{C} 4-\mathrm{C} 7-\mathrm{C} 8=$ $-62.8(2)^{\circ}$ and $\mathrm{C} 4-\mathrm{C} 7-\mathrm{C} 8-\mathrm{C} 9=152.2(2)^{\circ}$, thus exhibiting an extended conformation. The partially saturated $\mathrm{C} 1-\mathrm{C} 6$ ring adopts a sofa conformation. The distance of atom $\mathrm{C} 1$ from the mean plane formed by atoms $\mathrm{C} 2-\mathrm{C} 6$ is 0.612 (3) $\AA$. The dihedral angle between the mean plane of the $\mathrm{C} 1-\mathrm{C} 6$ ring and the $\mathrm{C} 8-\mathrm{C} 13$ benzene ring is $75.69(6)^{\circ}$.

\section{Supramolecular features}

In the crystal, the molecules are assembled into a sheet structure parallel to the $a b$ plane via $\mathrm{O}-\mathrm{H} \cdots \mathrm{O}$ hydrogen bonds (Table 1). The hydrogen-bonding pattern in the sheet is described by an $R_{4}^{4}(28)$ graph-set motif (Fig. 2). Furthermore, weak $\mathrm{C}-\mathrm{H} \cdots \mathrm{O}$ hydrogen bonds join the sheets into a threedimensional network (Table 1).

\section{Database survey}

A search of the Cambridge Structural Database (Version 5.39, last update February 2018; Groom et al., 2016) gave 76 structures of 3-hydroxy-5,5-dimethylcyclohex-2-enone derivatives. The closest structures are 2-(naphthalen-1-ylmethyl)and 2-(3-chlorophenyl)methyl-substituted dimedones

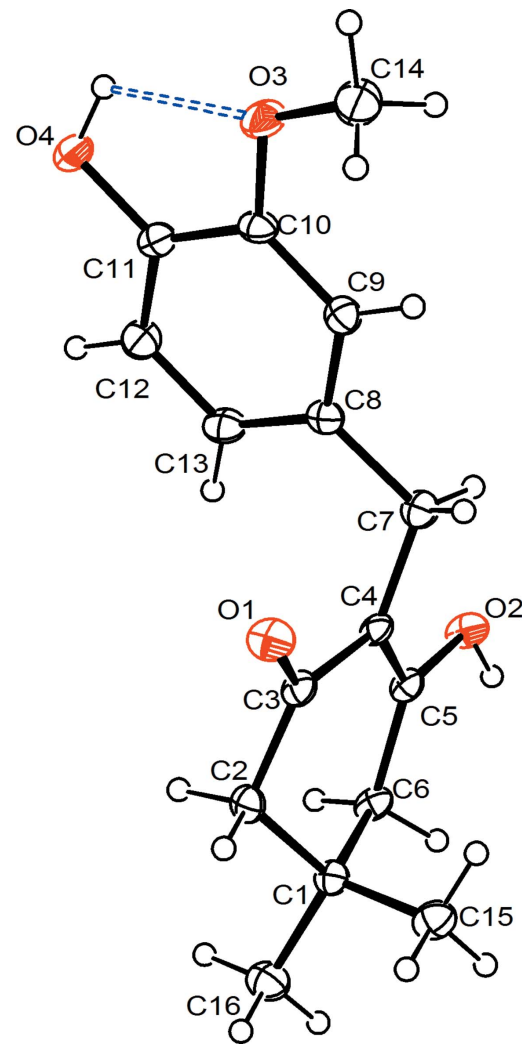

Figure 1

The molecular structure of the title compound, with the atom-numbering scheme and $50 \%$ probability displacement ellipsoids. The intramolecular hydrogen bond is shown as a double-dashed line.
Table 1

Hydrogen-bond geometry $\left(\AA{ }^{\circ}\right)$.

\begin{tabular}{lllll}
\hline$D-\mathrm{H} \cdots A$ & $D-\mathrm{H}$ & $\mathrm{H} \cdots A$ & $D \cdots A$ & $D-\mathrm{H} \cdots A$ \\
\hline $\mathrm{C} 2-\mathrm{H} 2 A \cdots \mathrm{O} 4^{\mathrm{i}}$ & 0.97 & 2.49 & $3.417(3)$ & 161 \\
$\mathrm{C} 14-\mathrm{H} 14 C \cdots \mathrm{O} 1^{\mathrm{ii}}$ & 0.96 & 2.49 & $3.247(3)$ & 136 \\
$\mathrm{O} 2-\mathrm{H} 2 \cdots \mathrm{O} 1^{\mathrm{iii}}$ & $0.88(3)$ & $1.74(3)$ & $2.586(2)$ & $161(3)$ \\
$\mathrm{O} 4-\mathrm{H} 4 \cdots \mathrm{O} 3$ & $0.94(4)$ & $2.10(4)$ & $2.638(2)$ & $115(3)$ \\
$\mathrm{O} 4-\mathrm{H} 4 \cdots \mathrm{O} 2^{\text {iv }}$ & $0.94(4)$ & $2.11(4)$ & $2.919(2)$ & $142(3)$ \\
\hline
\end{tabular}

Symmetry codes: (i) $-x+1,-y+1,-z+1$; (ii) $x-\frac{1}{2}, y,-z+\frac{3}{2}$; (iii) $-x+\frac{3}{2}, y+\frac{1}{2}, z$; (iv) $-x+\frac{1}{2}, y-\frac{1}{2}, z$.

(NIHTEE and NIHTII, respectively; Ramachary \& Kishor, 2007).

\section{Antiradical activity against free radicals}

Compound $\mathbf{1}$ demonstrates notable antiradical activity against free radicals. Free radical tests were realized according to the procedures described previously (Mierina et al., 2017). 1,1Diphenyl-2-picrylhydrazyl test: inhibition, when molar ratio of the compound and free radical is $1: 1$, was $93.3 \pm 2.5 \%$; $\mathrm{IC}_{50}$ was $23.0 \pm 0.6 \mu \mathrm{M}$ (starting concentration of free radical was $100 \mu \mathrm{M})$. Galvinoxyl test: inhibition was $82.3 \pm 1.0 \%$ and $\mathrm{IC}_{50}-$ $20.3 \pm 2.0 \mu \mathrm{M}$.

\section{Synthesis and crystallization}

3-Hydroxy-2-(4-hydroxy-3-methoxyphenylmethyl)-5,5-dimethylcyclohex-2-enone (1a) was synthesized according to the reaction scheme in Fig. 3. Formic acid $(3.6 \mathrm{ml})$ was added to a solution of dimedone 2 (500 mg, $3.6 \mathrm{mmol})$ and vanillin 3 $(543 \mathrm{mg}, 3.6 \mathrm{mmol})$ in triethylamine $(5.5 \mathrm{ml})$ while cooling in an ice-bath. The reaction mixture was then heated at $413 \mathrm{~K}$ for $5 \mathrm{~h}$, followed by cooling to room temperature, pouring into ice (700-800 ml) and filtering the formed solid. The solid material

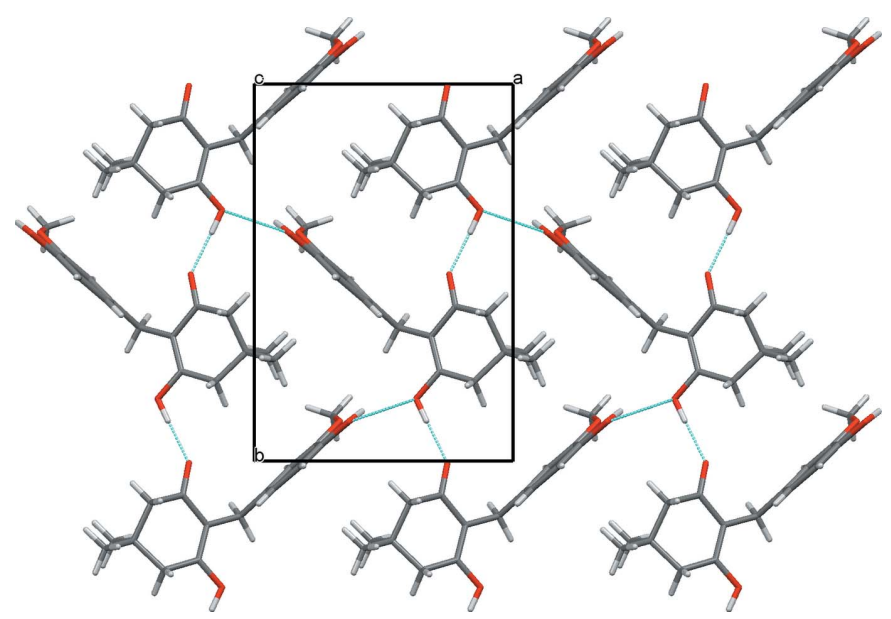

Figure 2

A packing diagram of the title compound, viewed along the $c$ axis. $\mathrm{O}-$ $\mathrm{H} \cdots \mathrm{O}$ hydrogen bonds are shown as dashed lines. For clarity weak C$\mathrm{H} \cdots \mathrm{O}$ bonds are not depicted. 
was purified by crystallization from chloroform leading to the target compound 1a (615 mg, 62\%) with m.p. 466-468 K. Single crystals were obtained from a methanol solution. IR (KBr) $v, \mathrm{~cm}^{-1}: 3470,2935,2645,1580,1515,1375,1250,1230$, 1200, 1040.

The enol form, 1a, was observed exclusively in a DMSO solution. ${ }^{1} \mathrm{H}$ NMR for compound $1 \mathbf{a}\left(300 \mathrm{MHz}, \mathrm{DMSO}-d_{6}\right) \delta$, ppm: 10.71-10.08 (1H, brs, OH), 8.68-8.37 (1H, brs, OH), 6.68 $\left(1 \mathrm{H}, s, \mathrm{H}^{\mathrm{Ar}}\right), 6.59\left(1 \mathrm{H}, d, J=7.7 \mathrm{~Hz}, \mathrm{H}^{\mathrm{Ar}}\right), 6.50(1 \mathrm{H}, d, J=$ $\left.7.7 \mathrm{~Hz}, \mathrm{H}^{\mathrm{Ar}}\right), 3.68(3 \mathrm{H}, s, \mathrm{OMe}), 3.41\left(2 \mathrm{H}\right.$, brs, $\mathrm{CH}_{2} \mathrm{Ar}$, overlapping with $\mathrm{H}_{2} \mathrm{O}$ signal), 2.34-2.13 (4H, brs, $\left.2 \mathrm{CH}_{2}\right), 0.98(6 \mathrm{H}$, $s, 2 \mathrm{Me}) \cdot{ }^{13} \mathrm{C}$ NMR for compound $1 \mathrm{a}\left(75 \mathrm{MHz}, \mathrm{DMSO}-d_{6}\right) \delta$, ppm: 147.1, 144.1, 132.7, 120.2, 115.0, 113.3, 112.5, 55.5, 31.7, 28.0, 26.5. Mixture of keto-enol tautomers (1a and 1b) was observed in a chloroform solution. The ratio of enol 1a and ketone 1b was 1.35:1 (at room temperature). ${ }^{1} \mathrm{H}$ NMR for compound 1a $\left(300 \mathrm{MHz}, \mathrm{CDCl}_{3}\right) \delta$, ppm: 6.84-6.63 $(3 \mathrm{H}, m$, $\left.\mathrm{H}^{\mathrm{Ar}}\right),(2 \mathrm{H}$, brs, $2 \mathrm{OH}), 3.82(3 \mathrm{H}, s, \mathrm{OMe}), 3.61\left(2 \mathrm{H}, s, \mathrm{CH}_{2} \mathrm{Ar}\right)$, 2.33-2.29 (4H, brs, $\left.2 \mathrm{CH}_{2}\right), 1.07(6 \mathrm{H}, s, 2 \mathrm{Me}) .{ }^{1} \mathrm{H} \mathrm{NMR}$ for compound $1 \mathbf{b}\left(300 \mathrm{MHz}, \mathrm{CDCl}_{3}\right) \delta$, ppm: 6.84-6.63 $(3 \mathrm{H}, m$, $\left.\mathrm{H}^{\mathrm{Ar}}\right), 5.62-5.68(1 \mathrm{H}$, brs, $\mathrm{OH}), 3.86(3 \mathrm{H}, s, \mathrm{OMe}), 3.56(1 \mathrm{H}, t, J$ $\left.=5.4 \mathrm{~Hz}, \mathrm{CHCH}_{2}\right), 3.11\left(2 \mathrm{H}, d, J=5.4 \mathrm{~Hz}, \mathrm{CHCH}_{2}\right), 2.65(2 \mathrm{H}$, $d, J=13.4 \mathrm{~Hz}, \mathrm{H}^{\mathrm{a}}$ from $\left.\mathrm{CH}_{2}\right), 2.44\left(2 \mathrm{H}, d, J=13.4 \mathrm{~Hz}, \mathrm{H}^{\mathrm{b}}\right.$ from $\left.\mathrm{CH}_{2}\right), 1.16(3 \mathrm{H}, s, \mathrm{Me}), 0.82(3 \mathrm{H}, \mathrm{s}, \mathrm{Me})$.

\section{Refinement}

Crystal data, data collection and structure refinement details are summarized in Table 2. Hydrogen atoms bonded to $\mathrm{O}$ atoms were refined freely. Other $\mathrm{H}$ atoms were included in the refinement at geometrically calculated positions with $\mathrm{C}-\mathrm{H}=$ $0.93-0.97 \AA$ and treated as riding with $U_{\text {iso }}(\mathrm{H})=1.2 U_{\text {eq }}(\mathrm{C})$ or $1.5 U_{\text {eq }}(\mathrm{C}-\mathrm{methyl})$.<smiles>CC1(C)CC(=O)CC(=O)C1</smiles>

2<smiles>COc1cc(C=O)ccc1O</smiles>

3

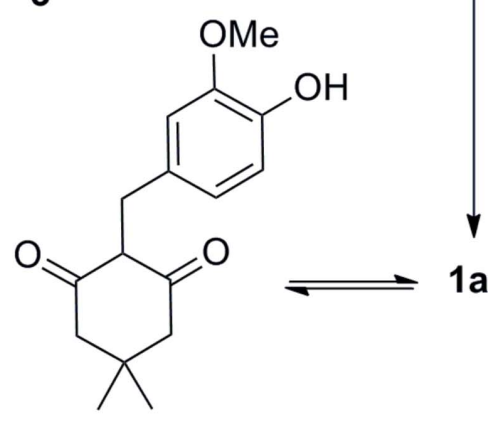

1b
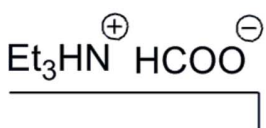

Figure 3

Reaction scheme for the title compound (1a) and its tautomer (1 b).
Table 2

Experimental details.

\begin{tabular}{ll}
\hline Crystal data & \\
Chemical formula & $\mathrm{C}_{16} \mathrm{H}_{20} \mathrm{O}_{4}$ \\
$M_{\mathrm{r}}$ & 276.32 \\
Crystal system, space group & Orthorhombic, Pbca \\
Temperature $(\mathrm{K})$ & 190 \\
$a, b, c(\AA)$ & $9.3504(3), 13.6265(4), 22.8790(9)$ \\
$V\left(\AA^{3}\right)$ & $2915.09(17)$ \\
$Z$ & 8 \\
Radiation type & Mo $K \alpha$ \\
$\mu\left(\mathrm{mm}^{-1}\right)$ & 0.09 \\
Crystal size $(\mathrm{mm})$ & $0.32 \times 0.17 \times 0.12$ \\
& \\
Data collection & \\
Diffractometer & $\mathrm{Bruker} \mathrm{KappaCCD}$ \\
No. of measured, independent and & $6082,3295,2149$ \\
$\quad$ observed $[I>2 \sigma(I)]$ reflections & \\
$R_{\text {int }}$ & 0.057 \\
$(\text { sin } \theta / \lambda)_{\text {max }}\left(\AA^{-1}\right)$ & 0.649 \\
& \\
Refinement & \\
$R\left[F^{2}>2 \sigma\left(F^{2}\right)\right], w R\left(F^{2}\right), S$ & $0.059,0.131,1.04$ \\
No. of reflections & 3295 \\
No. of parameters & 192 \\
$\mathrm{H}$-atom treatment & $\mathrm{H}$ atoms treated by a mixture of \\
& independent and constrained \\
$\Delta \rho_{\text {max }}, \Delta \rho_{\text {min }}\left(\mathrm{e} \AA^{-3}\right)$ & refinement \\
\hline
\end{tabular}

Computer programs: COLLECT (Bruker, 2001), SCALEPACK (Otwinowski \& Minor 1997), DENZO (Otwinowski \& Minor, 1997), SIR2004 (Burla et al., 2005), SHELXL2017 (Sheldrick, 2015), ORTEP-3 for Windows (Farrugia, 2012) and publCIF (Westrip, 2010).

\section{Funding information}

Funding for this research was provided by: European Regional Development Fund, Operational Programme 'Growth and Employment' within the Activity 'Post-doctoral Research Aid' (grant No. 1.1.1.2/VIAA/1/16/039).

\section{References}

Allen, F. H., Kennard, O., Watson, D. G., Brammer, L., Orpen, A. G. \& Taylor, R. (1987). J. Chem. Soc. Perkin Trans. 2, pp. S1-19.

Bruker (2001). COLLECT. Bruker AXS Inc., Madison, Wisconsin, USA.

Burla, M. C., Caliandro, R., Camalli, M., Carrozzini, B., Cascarano, G. L., De Caro, L., Giacovazzo, C., Polidori, G. \& Spagna, R. (2005). J. Appl. Cryst. 38, 381-388.

Farrugia, L. J. (2012). J. Appl. Cryst. 45, 849-854.

Groom, C. R., Bruno, I. J., Lightfoot, M. P. \& Ward, S. C. (2016). Acta Cryst. B72, 171-179.

Mierina, I., Jure, M., Zeberga, S., Makareviciene, V., Zicane, D., Tetere, Z. \& Ravina, I. (2017). Eur. J. Lipid Sci. Technol. 119, 1700172 .

Otwinowski, Z. \& Minor, W. (1997). Methods in Enzymology, Vol. 276, Macromolecular Crystallography, Part A, edited by C. W. Carter Jr \& R. M. Sweet, pp. 307-326. New York: Academic Press.

Ramachary, D. B. \& Kishor, M. (2007). J. Org. Chem. 72, 5056-5068.

Sheldrick, G. M. (2015). Acta Cryst. C71, 3-8.

Sudheendran, K., Malakar, C. C., Conrad, J. \& Beifuss, U. (2012). J. Org. Chem. 77, 10194-10210.

Westrip, S. P. (2010). J. Appl. Cryst. 43, 920-925.

Xu, C., Zhang, L., Zhou, P., Luo, S. \& Cheng, J.-P. (2013). Synthesis, 45, 1939-1945.

Yoshida, M., Higuchi, M. \& Shishido, K. (2010). Tetrahedron, 66, 2675-2682. 


\section{supporting information}

Acta Cryst. (2018). E74, 796-798 [https://doi.org/10.1107/S2056989018006941]

\section{Crystal structure of 3-hydroxy-2-(4-hydroxy-3-methoxyphenylmethyl)-5,5-di- methylcyclohex-2-enone}

\section{Agnese Stikute, Karina Skestere, Inese Mierina, Anatoly Mishnev and Mara Jure}

Computing details

Data collection: COLLECT (Bruker, 2001); cell refinement: SCALEPACK (Otwinowski \& Minor, 1997); data reduction: DENZO (Otwinowski \& Minor, 1997); program(s) used to solve structure: SIR2004 (Burla et al., 2005); program(s) used to refine structure: SHELXL2017 (Sheldrick, 2015); molecular graphics: ORTEP-3 for Windows (Farrugia, 2012); software used to prepare material for publication: publCIF (Westrip, 2010).

3-Hydroxy-2-(4-hydroxy-3-methoxyphenylmethyl)-5,5-dimethylcyclohex-2-enone

Crystal data

$\mathrm{C}_{16} \mathrm{H}_{20} \mathrm{O}_{4}$

$M_{r}=276.32$

Orthorhombic, $\mathrm{Pbca}$

$a=9.3504$ (3) $\AA$

$b=13.6265$ (4) $\AA$

$c=22.8790(9) \AA$

$V=2915.09(17) \AA^{3}$

$Z=8$

$F(000)=1184$

Data collection

Bruker KappaCCD

diffractometer

CCD scans

6082 measured reflections

3295 independent reflections

2149 reflections with $I>2 \sigma(I)$

\section{Refinement}

Refinement on $F^{2}$

Least-squares matrix: full

$R\left[F^{2}>2 \sigma\left(F^{2}\right)\right]=0.059$

$w R\left(F^{2}\right)=0.131$

$S=1.04$

3295 reflections

192 parameters

0 restraints
$D_{\mathrm{x}}=1.259 \mathrm{Mg} \mathrm{m}^{-3}$

Mo $K \alpha$ radiation, $\lambda=0.71073 \AA$

Cell parameters from 32401 reflections

$\theta=1.0-27.5^{\circ}$

$\mu=0.09 \mathrm{~mm}^{-1}$

$T=190 \mathrm{~K}$

Block, colourless

$0.32 \times 0.17 \times 0.12 \mathrm{~mm}$

$R_{\text {int }}=0.057$

$\theta_{\max }=27.5^{\circ}, \theta_{\min }=2.8^{\circ}$

$h=-12 \rightarrow 12$

$k=-17 \rightarrow 17$

$l=-29 \rightarrow 29$

Hydrogen site location: mixed

$\mathrm{H}$ atoms treated by a mixture of independent and constrained refinement

$w=1 /\left[\sigma^{2}\left(F_{\mathrm{o}}^{2}\right)+(0.0489 P)^{2}+1.0327 P\right]$

where $P=\left(F_{\mathrm{o}}{ }^{2}+2 F_{\mathrm{c}}{ }^{2}\right) / 3$

$(\Delta / \sigma)_{\max }=0.006$

$\Delta \rho_{\max }=0.21 \mathrm{e} \AA^{-3}$

$\Delta \rho_{\min }=-0.19$ e $\AA^{-3}$ 


\section{Special details}

Geometry. All esds (except the esd in the dihedral angle between two 1.s. planes) are estimated using the full covariance matrix. The cell esds are taken into account individually in the estimation of esds in distances, angles and torsion angles; correlations between esds in cell parameters are only used when they are defined by crystal symmetry. An approximate (isotropic) treatment of cell esds is used for estimating esds involving l.s. planes.

Fractional atomic coordinates and isotropic or equivalent isotropic displacement parameters $\left(\hat{A}^{2}\right)$

\begin{tabular}{|c|c|c|c|c|}
\hline & $x$ & $y$ & $z$ & $U_{\text {iso }} * / U_{\text {eq }}$ \\
\hline $\mathrm{O} 1$ & $0.75371(16)$ & $0.50507(9)$ & $0.63597(7)$ & $0.0331(4)$ \\
\hline $\mathrm{O} 2$ & $0.62541(15)$ & $0.83466(10)$ & $0.63025(7)$ & $0.0287(4)$ \\
\hline $\mathrm{O} 3$ & $0.16966(16)$ & $0.39470(10)$ & $0.68561(7)$ & $0.0367(4)$ \\
\hline $\mathrm{O} 4$ & $0.13650(15)$ & $0.39579(10)$ & $0.57111(7)$ & $0.0319(4)$ \\
\hline $\mathrm{C} 1$ & $0.9683(2)$ & $0.71070(12)$ & $0.58699(9)$ & $0.0224(4)$ \\
\hline $\mathrm{C} 2$ & $0.8992(2)$ & $0.60988(13)$ & $0.57748(9)$ & $0.0256(5)$ \\
\hline $\mathrm{H} 2 \mathrm{~A}$ & 0.864618 & 0.605845 & 0.537577 & $0.031^{*}$ \\
\hline $\mathrm{H} 2 \mathrm{~B}$ & 0.971321 & 0.559458 & 0.582589 & $0.031^{*}$ \\
\hline $\mathrm{C} 3$ & $0.7777(2)$ & $0.58996(13)$ & $0.61829(9)$ & $0.0230(5)$ \\
\hline $\mathrm{C} 4$ & $0.6838(2)$ & $0.66925(13)$ & $0.63552(9)$ & $0.0213(4)$ \\
\hline $\mathrm{C} 5$ & $0.7173(2)$ & $0.76168(13)$ & $0.61827(9)$ & $0.0216(4)$ \\
\hline C6 & $0.8495(2)$ & $0.78837(13)$ & $0.58489(9)$ & $0.0243(5)$ \\
\hline H6A & 0.886941 & 0.849470 & 0.600375 & $0.029^{*}$ \\
\hline H6B & 0.823687 & 0.799694 & 0.544389 & $0.029^{*}$ \\
\hline $\mathrm{C} 7$ & $0.5558(2)$ & $0.64569(13)$ & $0.67303(10)$ & $0.0277(5)$ \\
\hline H7A & 0.508367 & 0.706600 & 0.683286 & $0.033^{*}$ \\
\hline H7B & 0.589063 & 0.615710 & 0.709003 & $0.033^{*}$ \\
\hline $\mathrm{C} 8$ & $0.4470(2)$ & $0.57763(13)$ & $0.64472(9)$ & $0.0232(5)$ \\
\hline C9 & $0.3616(2)$ & $0.51732(13)$ & $0.68030(9)$ & $0.0258(5)$ \\
\hline H9 & 0.374309 & 0.517992 & 0.720617 & $0.031^{*}$ \\
\hline $\mathrm{C} 10$ & $0.2589(2)$ & $0.45705(13)$ & $0.65589(9)$ & $0.0252(5)$ \\
\hline C11 & $0.2391(2)$ & $0.45584(13)$ & $0.59560(9)$ & $0.0244(5)$ \\
\hline $\mathrm{C} 12$ & $0.3230(2)$ & $0.51344(15)$ & $0.56037(10)$ & $0.0300(5)$ \\
\hline H12 & 0.310764 & 0.512153 & 0.520037 & $0.036^{*}$ \\
\hline $\mathrm{C} 13$ & $0.4265(2)$ & $0.57391(14)$ & $0.58529(10)$ & $0.0287(5)$ \\
\hline H13 & 0.483160 & 0.612663 & 0.561127 & $0.034^{*}$ \\
\hline $\mathrm{C} 14$ & $0.1966(3)$ & $0.37943(17)$ & $0.74571(11)$ & $0.0469(7)$ \\
\hline H14A & 0.293311 & 0.357396 & 0.750859 & $0.070^{*}$ \\
\hline H14B & 0.131964 & 0.330679 & 0.760536 & $0.070^{*}$ \\
\hline $\mathrm{H} 14 \mathrm{C}$ & 0.182851 & 0.439830 & 0.766563 & $0.070^{*}$ \\
\hline $\mathrm{C} 15$ & $1.0448(2)$ & $0.71292(14)$ & $0.64588(10)$ & $0.0317(5)$ \\
\hline $\mathrm{H} 15 \mathrm{~A}$ & 0.977325 & 0.700158 & 0.676564 & $0.048^{*}$ \\
\hline H15B & 1.087200 & 0.776352 & 0.651680 & $0.048^{*}$ \\
\hline $\mathrm{H} 15 \mathrm{C}$ & 1.118201 & 0.663631 & 0.646450 & $0.048^{*}$ \\
\hline C16 & $1.0760(2)$ & $0.73067(15)$ & $0.53816(10)$ & $0.0345(5)$ \\
\hline H16A & 1.115861 & 0.795077 & 0.543051 & $0.052^{*}$ \\
\hline H16B & 1.028672 & 0.726735 & 0.500998 & $0.052^{*}$ \\
\hline H16C & 1.151155 & 0.682745 & 0.539763 & $0.052^{*}$ \\
\hline $\mathrm{H} 2$ & $0.664(3)$ & $0.892(2)$ & $0.6238(13)$ & $0.079(10)^{*}$ \\
\hline
\end{tabular}


Atomic displacement parameters $\left(\AA^{2}\right)$

\begin{tabular}{lllllll}
\hline & $U^{11}$ & $U^{22}$ & $U^{33}$ & $U^{12}$ & $U^{13}$ & $U^{23}$ \\
\hline O1 & $0.0372(8)$ & $0.0110(6)$ & $0.0510(10)$ & $-0.0009(6)$ & $0.0078(8)$ & $0.0033(6)$ \\
O2 & $0.0240(8)$ & $0.0128(7)$ & $0.0493(10)$ & $0.0022(6)$ & $-0.0014(7)$ & $0.0001(6)$ \\
O3 & $0.0393(9)$ & $0.0406(9)$ & $0.0304(9)$ & $-0.0196(7)$ & $0.0011(7)$ & $0.0042(7)$ \\
O4 & $0.0283(8)$ & $0.0362(8)$ & $0.0312(9)$ & $-0.0096(7)$ & $-0.0035(7)$ & $-0.0033(7)$ \\
C1 & $0.0241(10)$ & $0.0168(9)$ & $0.0262(11)$ & $-0.0004(8)$ & $0.0006(9)$ & $0.0004(8)$ \\
C2 & $0.0287(11)$ & $0.0172(9)$ & $0.0308(12)$ & $0.0006(8)$ & $0.0033(9)$ & $-0.0035(8)$ \\
C3 & $0.0253(11)$ & $0.0149(9)$ & $0.0288(12)$ & $-0.0019(8)$ & $-0.0012(9)$ & $-0.0016(8)$ \\
C4 & $0.0199(10)$ & $0.0145(9)$ & $0.0297(12)$ & $-0.0023(8)$ & $-0.0019(9)$ & $-0.0035(8)$ \\
C5 & $0.0207(11)$ & $0.0163(9)$ & $0.0278(12)$ & $0.0010(8)$ & $-0.0047(8)$ & $-0.0014(8)$ \\
C6 & $0.0241(10)$ & $0.0151(9)$ & $0.0336(12)$ & $-0.0028(8)$ & $-0.0038(9)$ & $0.0029(8)$ \\
C7 & $0.0306(12)$ & $0.0160(9)$ & $0.0366(14)$ & $-0.0026(9)$ & $0.0042(10)$ & $-0.0034(8)$ \\
C8 & $0.0222(10)$ & $0.0164(9)$ & $0.0310(12)$ & $0.0031(8)$ & $0.0031(9)$ & $0.0003(8)$ \\
C9 & $0.0283(11)$ & $0.0233(10)$ & $0.0260(12)$ & $0.0005(9)$ & $0.0014(9)$ & $0.0000(8)$ \\
C10 & $0.0236(10)$ & $0.0198(9)$ & $0.0322(12)$ & $-0.0023(9)$ & $0.0060(9)$ & $0.0024(8)$ \\
C11 & $0.0207(10)$ & $0.0197(9)$ & $0.0328(12)$ & $0.0011(8)$ & $-0.0013(9)$ & $-0.0003(9)$ \\
C12 & $0.0330(12)$ & $0.0300(11)$ & $0.0271(13)$ & $-0.0028(10)$ & $-0.0005(10)$ & $0.0037(9)$ \\
C13 & $0.0279(11)$ & $0.0222(10)$ & $0.0359(13)$ & $-0.0035(9)$ & $0.0038(10)$ & $0.0061(9)$ \\
C14 & $0.0604(17)$ & $0.0503(14)$ & $0.0300(14)$ & $-0.0262(13)$ & $0.0077(12)$ & $0.0032(11)$ \\
C15 & $0.0263(11)$ & $0.0273(11)$ & $0.0414(14)$ & $0.0024(9)$ & $-0.0045(10)$ & $-0.0003(10)$ \\
C16 & $0.0333(12)$ & $0.0270(11)$ & $0.0432(15)$ & $-0.0024(10)$ & $0.0077(10)$ & $0.0000(10)$ \\
& & & & & &
\end{tabular}

Geometric parameters $\left(\AA,{ }^{\circ}\right)$

\begin{tabular}{llll}
\hline $\mathrm{O} 1-\mathrm{C} 3$ & $1.246(2)$ & $\mathrm{C} 7-\mathrm{H} 7 \mathrm{~A}$ & 0.9700 \\
$\mathrm{O} 2-\mathrm{C} 5$ & $1.342(2)$ & $\mathrm{C} 7-\mathrm{H} 7 \mathrm{~B}$ & 0.9700 \\
$\mathrm{O} 2-\mathrm{H} 2$ & $0.88(3)$ & $\mathrm{C} 8-\mathrm{C} 13$ & $1.374(3)$ \\
$\mathrm{O} 3-\mathrm{C} 10$ & $1.371(2)$ & $\mathrm{C} 8-\mathrm{C} 9$ & $1.381(3)$ \\
$\mathrm{O} 3-\mathrm{C} 14$ & $1.413(3)$ & $\mathrm{C} 9-\mathrm{C} 10$ & 0.9300 \\
$\mathrm{O} 4-\mathrm{C} 11$ & $1.380(2)$ & $\mathrm{C} 9-\mathrm{H} 9$ & $1.392(3)$ \\
$\mathrm{O} 4-\mathrm{H} 4$ & $0.94(4)$ & $\mathrm{C} 10-\mathrm{C} 11$ & $1.371(3)$ \\
$\mathrm{C} 1-\mathrm{C} 15$ & $1.526(3)$ & $\mathrm{C} 11-\mathrm{C} 12$ & $1.394(3)$ \\
$\mathrm{C} 1-\mathrm{C} 16$ & $1.529(3)$ & $\mathrm{C} 12-\mathrm{C} 13$ & 0.9300 \\
$\mathrm{C} 1-\mathrm{C} 2$ & $1.534(3)$ & $\mathrm{C} 12-\mathrm{H} 12$ & 0.9300 \\
$\mathrm{C} 1-\mathrm{C} 6$ & $1.534(3)$ & $\mathrm{C} 13-\mathrm{H} 13$ & 0.9600 \\
$\mathrm{C} 2-\mathrm{C} 3$ & $1.495(3)$ & $\mathrm{C} 14-\mathrm{H} 14 \mathrm{~A}$ & 0.9600 \\
$\mathrm{C} 2-\mathrm{H} 2 \mathrm{~A}$ & $\mathrm{C} 14-\mathrm{H} 14 \mathrm{~B}$ & 0.9600 \\
$\mathrm{C} 2-\mathrm{H} 2 \mathrm{~B}$ & 0.9700 & $\mathrm{C} 14-\mathrm{H} 14 \mathrm{C}$ & 0.9600 \\
$\mathrm{C} 3-\mathrm{C} 4$ & 0.9700 & $\mathrm{C} 15-\mathrm{H} 15 \mathrm{~A}$ & 0.9600 \\
$\mathrm{C} 4-\mathrm{C} 5$ & $1.447(3)$ & $\mathrm{C} 15-\mathrm{H} 15 \mathrm{~B}$ & 0.9600 \\
$\mathrm{C} 4-\mathrm{C} 7$ & $1.357(3)$ & $\mathrm{C} 15-\mathrm{H} 15 \mathrm{C}$ & 0.9600 \\
$\mathrm{C} 5-\mathrm{C} 6$ & $1.507(3)$ & $\mathrm{C} 16-\mathrm{H} 16 \mathrm{~A}$ & 0.9600 \\
$\mathrm{C} 6-\mathrm{H} 6 \mathrm{~A}$ & $1.498(3)$ & $\mathrm{C} 16-\mathrm{H} 16 \mathrm{~B}$ & 0.9600 \\
$\mathrm{C} 6-\mathrm{H} 6 \mathrm{~B}$ & 0.9700 & $\mathrm{C} 16-\mathrm{H} 16 \mathrm{C}$ &
\end{tabular}


$\mathrm{C} 7-\mathrm{C} 8$

$\mathrm{C} 5-\mathrm{O} 2-\mathrm{H} 2$

$\mathrm{C} 10-\mathrm{O} 3-\mathrm{C} 14$

$\mathrm{C} 11-\mathrm{O} 4-\mathrm{H} 4$

$\mathrm{C} 15-\mathrm{C} 1-\mathrm{C} 16$

$\mathrm{C} 15-\mathrm{C} 1-\mathrm{C} 2$

$\mathrm{C} 16-\mathrm{C} 1-\mathrm{C} 2$

$\mathrm{C} 15-\mathrm{C} 1-\mathrm{C} 6$

$\mathrm{C} 16-\mathrm{C} 1-\mathrm{C} 6$

$\mathrm{C} 2-\mathrm{C} 1-\mathrm{C} 6$

$\mathrm{C} 3-\mathrm{C} 2-\mathrm{C} 1$

$\mathrm{C} 3-\mathrm{C} 2-\mathrm{H} 2 \mathrm{~A}$

$\mathrm{C} 1-\mathrm{C} 2-\mathrm{H} 2 \mathrm{~A}$

$\mathrm{C} 3-\mathrm{C} 2-\mathrm{H} 2 \mathrm{~B}$

$\mathrm{C} 1-\mathrm{C} 2-\mathrm{H} 2 \mathrm{~B}$

$\mathrm{H} 2 \mathrm{~A}-\mathrm{C} 2-\mathrm{H} 2 \mathrm{~B}$

$\mathrm{O} 1-\mathrm{C} 3-\mathrm{C} 4$

$\mathrm{O} 1-\mathrm{C} 3-\mathrm{C} 2$

$\mathrm{C} 4-\mathrm{C} 3-\mathrm{C} 2$

$\mathrm{C} 5-\mathrm{C} 4-\mathrm{C} 3$

$\mathrm{C} 5-\mathrm{C} 4-\mathrm{C} 7$

$\mathrm{C} 3-\mathrm{C} 4-\mathrm{C} 7$

$\mathrm{O} 2-\mathrm{C} 5-\mathrm{C} 4$

$\mathrm{O} 2-\mathrm{C} 5-\mathrm{C} 6$

$\mathrm{C} 4-\mathrm{C} 5-\mathrm{C} 6$

$\mathrm{C} 5-\mathrm{C} 6-\mathrm{C} 1$

C5-C6-H6A

$\mathrm{C} 1-\mathrm{C} 6-\mathrm{H} 6 \mathrm{~A}$

C5- 6 - $-\mathrm{H} 6 \mathrm{~B}$

$\mathrm{C} 1-\mathrm{C} 6-\mathrm{H} 6 \mathrm{~B}$

$\mathrm{H} 6 \mathrm{~A}-\mathrm{C} 6-\mathrm{H} 6 \mathrm{~B}$

$\mathrm{C} 4-\mathrm{C} 7-\mathrm{C} 8$

$\mathrm{C} 4-\mathrm{C} 7-\mathrm{H} 7 \mathrm{~A}$

$\mathrm{C} 8-\mathrm{C} 7-\mathrm{H} 7 \mathrm{~A}$

$\mathrm{C} 4-\mathrm{C} 7-\mathrm{H} 7 \mathrm{~B}$

$\mathrm{C} 8-\mathrm{C} 7-\mathrm{H} 7 \mathrm{~B}$

$\mathrm{H} 7 \mathrm{~A}-\mathrm{C} 7-\mathrm{H} 7 \mathrm{~B}$

$\mathrm{C} 15-\mathrm{C} 1-\mathrm{C} 2-\mathrm{C} 3$

$\mathrm{C} 16-\mathrm{C} 1-\mathrm{C} 2-\mathrm{C} 3$

$\mathrm{C} 6-\mathrm{C} 1-\mathrm{C} 2-\mathrm{C} 3$

$\mathrm{C} 1-\mathrm{C} 2-\mathrm{C} 3-\mathrm{O} 1$

$\mathrm{C} 1-\mathrm{C} 2-\mathrm{C} 3-\mathrm{C} 4$

$\mathrm{O} 1-\mathrm{C} 3-\mathrm{C} 4-\mathrm{C} 5$

$\mathrm{C} 2-\mathrm{C} 3-\mathrm{C} 4-\mathrm{C} 5$

$\mathrm{O} 1-\mathrm{C} 3-\mathrm{C} 4-\mathrm{C} 7$

$\mathrm{C} 2-\mathrm{C} 3-\mathrm{C} 4-\mathrm{C} 7$
$1.521(3)$

111 (2)

$117.73(17)$

107 (2)

$109.41(17)$

$109.91(16)$

$109.48(16)$

$110.69(16)$

$109.34(16)$

$107.99(16)$

$113.20(15)$

108.9

108.9

108.9

108.9

107.8

$119.70(18)$

$120.54(17)$

$119.71(16)$

$118.27(18)$

$123.16(17)$

$118.55(16)$

$118.71(17)$

$116.90(15)$

$124.37(17)$

$114.44(15)$

108.7

108.7

108.7

108.7

107.6

114.73 (17)

108.6

108.6

108.6

108.6

107.6

$67.9(2)$

$-171.93(17)$

$-53.0(2)$

-146.67 (19)

35.9 (3)

176.34 (19)

$-6.2(3)$

$-2.0(3)$

175.47 (18)
$\mathrm{C} 13-\mathrm{C} 8-\mathrm{C} 9$

$\mathrm{C} 13-\mathrm{C} 8-\mathrm{C} 7$

$\mathrm{C} 9-\mathrm{C} 8-\mathrm{C} 7$

$\mathrm{C} 10-\mathrm{C} 9-\mathrm{C} 8$

$\mathrm{C} 10-\mathrm{C} 9-\mathrm{H} 9$

C8- $99-\mathrm{H} 9$

$\mathrm{O} 3-\mathrm{C} 10-\mathrm{C} 9$

$\mathrm{O} 3-\mathrm{C} 10-\mathrm{C} 11$

C9- $\mathrm{C} 10-\mathrm{C} 11$

$\mathrm{C} 12-\mathrm{C} 11-\mathrm{O} 4$

$\mathrm{C} 12-\mathrm{C} 11-\mathrm{C} 10$

$\mathrm{O} 4-\mathrm{C} 11-\mathrm{C} 10$

$\mathrm{C} 11-\mathrm{C} 12-\mathrm{C} 13$

$\mathrm{C} 11-\mathrm{C} 12-\mathrm{H} 12$

$\mathrm{C} 13-\mathrm{C} 12-\mathrm{H} 12$

$\mathrm{C} 8-\mathrm{C} 13-\mathrm{C} 12$

$\mathrm{C} 8-\mathrm{C} 13-\mathrm{H} 13$

$\mathrm{C} 12-\mathrm{C} 13-\mathrm{H} 13$

O3- $14-\mathrm{H} 14 \mathrm{~A}$

O3- $14-\mathrm{H} 14 \mathrm{~B}$

H14A-C14-H14B

$\mathrm{O} 3-\mathrm{C} 14-\mathrm{H} 14 \mathrm{C}$

$\mathrm{H} 14 \mathrm{~A}-\mathrm{C} 14-\mathrm{H} 14 \mathrm{C}$

H14B - C14-H14C

C1-C15-H15A

C1-C15-H15B

$\mathrm{H} 15 \mathrm{~A}-\mathrm{C} 15-\mathrm{H} 15 \mathrm{~B}$

$\mathrm{C} 1-\mathrm{C} 15-\mathrm{H} 15 \mathrm{C}$

$\mathrm{H} 15 \mathrm{~A}-\mathrm{C} 15-\mathrm{H} 15 \mathrm{C}$

$\mathrm{H} 15 \mathrm{~B}-\mathrm{C} 15-\mathrm{H} 15 \mathrm{C}$

C1-C16-H16A

$\mathrm{C} 1-\mathrm{C} 16-\mathrm{H} 16 \mathrm{~B}$

H16A-C16-H16B

$\mathrm{C} 1-\mathrm{C} 16-\mathrm{H} 16 \mathrm{C}$

$\mathrm{H} 16 \mathrm{~A}-\mathrm{C} 16-\mathrm{H} 16 \mathrm{C}$

$\mathrm{H} 16 \mathrm{~B}-\mathrm{C} 16-\mathrm{H} 16 \mathrm{C}$

$\mathrm{C} 3-\mathrm{C} 4-\mathrm{C} 7-\mathrm{C} 8$

$\mathrm{C} 4-\mathrm{C} 7-\mathrm{C} 8-\mathrm{C} 13$

$\mathrm{C} 4-\mathrm{C} 7-\mathrm{C} 8-\mathrm{C} 9$

$\mathrm{C} 13-\mathrm{C} 8-\mathrm{C} 9-\mathrm{C} 10$

$\mathrm{C} 7-\mathrm{C} 8-\mathrm{C} 9-\mathrm{C} 10$

$\mathrm{C} 14-\mathrm{O} 3-\mathrm{C} 10-\mathrm{C} 9$

$\mathrm{C} 14-\mathrm{O} 3-\mathrm{C} 10-\mathrm{C} 11$

$\mathrm{C} 8-\mathrm{C} 9-\mathrm{C} 10-\mathrm{O} 3$

$\mathrm{C} 8-\mathrm{C} 9-\mathrm{C} 10-\mathrm{C} 11$
$118.18(18)$

122.47 (18)

119.33 (19)

120.5 (2)

119.7

119.7

$126.20(19)$

$113.78(17)$

$120.02(18)$

119.9 (2)

119.98 (19)

120.14 (18)

119.7 (2)

120.2

120.2

121.57 (19)

119.2

119.2

109.5

109.5

109.5

109.5

109.5

109.5

109.5

109.5

109.5

109.5

109.5

109.5

109.5

109.5

109.5

109.5

109.5

109.5

$-62.8(2)$

-29.1 (3)

152.22 (17)

-0.8 (3)

177.99 (17)

$-9.4(3)$

170.34 (19)

179.63 (18)

-0.1 (3) 


$\begin{array}{llll}\mathrm{C} 3-\mathrm{C} 4-\mathrm{C} 5-\mathrm{O} 2 & 175.08(17) & \mathrm{O} 3-\mathrm{C} 10-\mathrm{C} 11-\mathrm{C} 12 & -178.86(17) \\ \mathrm{C} 7-\mathrm{C} 4-\mathrm{C} 5-\mathrm{O} 2 & -6.6(3) & \mathrm{C} 9-\mathrm{C} 10-\mathrm{C} 11-\mathrm{C} 12 & 0.9(3) \\ \mathrm{C} 3-\mathrm{C} 4-\mathrm{C} 5-\mathrm{C} 6 & -3.2(3) & \mathrm{O} 3-\mathrm{C} 10-\mathrm{C} 11-\mathrm{O} 4 & 0.3(3) \\ \mathrm{C} 7-\mathrm{C} 4-\mathrm{C} 5-\mathrm{C} 6 & 175.11(19) & \mathrm{O} 4-\mathrm{C} 11-\mathrm{C} 12-\mathrm{C} 13 & -179.93(16) \\ \mathrm{O} 2-\mathrm{C} 5-\mathrm{C} 6-\mathrm{C} 1 & 163.97(17) & \mathrm{C} 10-\mathrm{C} 11-\mathrm{C} 12-\mathrm{C} 13 & -0.8(3) \\ \mathrm{C} 4-\mathrm{C} 5-\mathrm{C} 6-\mathrm{C} 1 & -17.7(3) & \mathrm{C} 9-\mathrm{C} 8-\mathrm{C} 13-\mathrm{C} 12 & 0.9(3) \\ \mathrm{C} 15-\mathrm{C} 1-\mathrm{C} 6-\mathrm{C} 5 & -76.1(2) & \mathrm{C} 7-\mathrm{C} 8-\mathrm{C} 13-\mathrm{C} 12 & -177.81(18) \\ \mathrm{C} 16-\mathrm{C} 1-\mathrm{C} 6-\mathrm{C} 5 & 163.26(17) & \mathrm{C} 11-\mathrm{C} 12-\mathrm{C} 13-\mathrm{C} 8 & -0.1(3) \\ \mathrm{C} 2-\mathrm{C} 1-\mathrm{C} 6-\mathrm{C} 5 & 44.2(2) & & \\ \mathrm{C} 5-\mathrm{C} 4-\mathrm{C} 7-\mathrm{C} 8 & 118.9(2) & & \end{array}$

Hydrogen-bond geometry $\left(\AA,{ }^{\circ}\right)$

\begin{tabular}{lllll}
\hline$D-\mathrm{H} \cdots A$ & $D-\mathrm{H}$ & $\mathrm{H} \cdots A$ & $D \cdots A$ & $D-\mathrm{H} \cdots A$ \\
\hline $\mathrm{C} 2-\mathrm{H} 2 A \cdots \mathrm{O} 4^{\mathrm{i}}$ & 0.97 & 2.49 & $3.417(3)$ & 161 \\
$\mathrm{C} 14-\mathrm{H} 14 C \cdots \mathrm{O} 1^{\mathrm{ii}}$ & 0.96 & 2.49 & $3.247(3)$ & 136 \\
$\mathrm{O} 2-\mathrm{H} 2 \cdots \mathrm{O} 1^{\mathrm{iii}}$ & $0.88(3)$ & $1.74(3)$ & $2.586(2)$ & $161(3)$ \\
$\mathrm{O} 4-\mathrm{H} 4 \cdots \mathrm{O} 3$ & $0.94(4)$ & $2.10(4)$ & $2.638(2)$ & $115(3)$ \\
$\mathrm{O} 4-\mathrm{H} 4 \cdots \mathrm{O} 2^{\text {iv }}$ & $0.94(4)$ & $2.11(4)$ & $2.919(2)$ & $142(3)$ \\
\hline
\end{tabular}

Symmetry codes: (i) $-x+1,-y+1,-z+1$; (ii) $x-1 / 2, y,-z+3 / 2$; (iii) $-x+3 / 2, y+1 / 2, z$; (iv) $-x+1 / 2, y-1 / 2, z$. 